DESY 10-128

August 2010

\title{
Matter and Dark Matter from False Vacuum Decay
}

\author{
W. Buchmüller, K. Schmitz, G. Vertongen \\ Deutsches Elektronen-Synchrotron DESY, 22607 Hamburg, Germany
}

\begin{abstract}
We study tachyonic preheating associated with the spontaneous breaking of $B-L$, the difference of baryon and lepton number. Reheating occurs through the decays of heavy Majorana neutrinos which are produced during preheating and in decays of the Higgs particles of $B-L$ breaking. Baryogenesis is an interplay of nonthermal and thermal leptogenesis, accompanied by thermally produced gravitino dark matter. The proposed mechanism simultaneously explains the generation of matter and dark matter, thereby relating the absolute neutrino mass scale to the gravitino mass.
\end{abstract}




\section{Introduction}

Thermal leptogenesis [1] explains the observed matter-antimatter asymmetry of the universe in terms of neutrino masses that are consistent with neutrino oscillation experiments. In its simplest version, the primordial lepton asymmetry is generated by the $C P$ violating interactions of $N_{1}$, the lightest of the heavy Majorana neutrinos, the seesaw partners of the ordinary neutrinos. Typical values for the $N_{1}$ mass and for the leptogenesis temperature are $M_{1} \sim T_{L} \sim 10^{10} \mathrm{GeV}[2$.

In supersymmetric theories such high reheating temperatures cause the 'gravitino problem' for heavy unstable gravitinos [3,45]. However, if the gravitino is the lightest superparticle (LSP), this problem can become a virtue since for typical leptogenesis temperatures and for superparticle masses of the electroweak scale, thermal production of gravitinos can explain the observed amount of dark matter [6]. The dominant contribution from QCD processes is given by

$$
\Omega_{\tilde{G}} h^{2}=C\left(\frac{T_{R}}{10^{10} \mathrm{GeV}}\right)\left(\frac{100 \mathrm{GeV}}{m_{\tilde{G}}}\right)\left(\frac{m_{\tilde{g}}}{1 \mathrm{TeV}}\right)^{2},
$$

where $T_{R}$ is the reheating temperature while $m_{\tilde{G}}$ and $m_{\tilde{g}}$ are gravitino and gluino masses, respectively; the coefficient $C=0.27$ to leading order in the gauge coupling [78] 1 Hence, for $T_{R} \sim T_{L}$ and superparticle masses characteristic of gravity or gaugino mediation one indeed obtains the observed amount of dark matter, $\Omega_{\mathrm{DM}} h^{2} \simeq 0.11$ [10].

Thermal leptogenesis is consistent with a reheating temperature $T_{R}$ much larger than $T_{L}$, which is not the case for thermally produced gravitino dark matter. Consistency of these two mechanisms for the origin of matter and dark matter then requires an explanation of why $T_{L}$ and $T_{R}$ have the same order of magnitude.

Successful leptogenesis, independent of initial conditions, favours the mass window $10^{-3} \mathrm{eV} \lesssim m_{i} \lesssim 0.1 \mathrm{eV}[11$ ] for the light neutrino masses. For an effective neutrino mass $\widetilde{m}_{1}=\left(m_{D}^{\dagger} m_{D}\right)_{11} / M_{1} \sim 0.01 \mathrm{eV}$, where $m_{D}$ is the Dirac neutrino mass, the decay width of the heavy Majorana neutrino $N_{1}$ becomes

$$
\Gamma_{N_{1}}^{0}=\frac{\widetilde{m}_{1}}{8 \pi}\left(\frac{M_{1}}{v_{\mathrm{EW}}}\right)^{2} \sim 10^{3} \mathrm{GeV},
$$

where we have used $v_{\mathrm{EW}}=174 \mathrm{GeV}$ for the vacuum expectation value of electroweak symmetry breaking. If the hot phase of the early universe is initiated by the decays of

\footnotetext{
${ }^{1}$ Note that $C$ has an $\mathcal{O}(1)$ uncertainty due to unknown higher order contributions and nonperturbative effects [7]. Resummation of thermal masses increases $C$ by about a factor of two [9].
} 
particles with decay width $\Gamma$, the reheating temperature is given by

$$
T_{R}=\left(\frac{90}{8 \pi^{3} g_{*}}\right)^{1 / 4} \sqrt{\Gamma M_{P}},
$$

where $g_{*} \sim 200$ is the effective number of relativistic degrees of freedom and $M_{P}=$ $1.22 \times 10^{19} \mathrm{GeV}$ is the Planck mass. If reheating occurs through the decay of $N_{1}$ neutrinos, one obtains

$$
T_{R} \sim 0.2 \cdot \sqrt{\Gamma_{N_{1}}^{0} M_{P}} \sim 10^{10} \mathrm{GeV}
$$

which is indeed the temperature needed for thermal leptogenesis and gravitino dark matter.

This observation raises the question whether in the thermal bath produced by heavy Majorana neutrino decays both, the matter-antimatter asymmetry and gravitino dark matter, can be simultaneously produced. In the following we shall present an example which demonstrates that this is indeed possible.

\section{Flavour model and tachyonic preheating}

In supersymmetric extensions of the Standard Model the superpotential for matter fields including right-handed neutrinos reads

$$
W_{M}=h_{i j}^{u} \mathbf{1 0}_{i} \mathbf{1 0}_{j} H_{u}+h_{i j}^{d} \mathbf{5}_{i}^{*} \mathbf{1 0}_{j} H_{d}+h_{i j}^{\nu} \mathbf{5}_{i}^{*} n_{j}^{c} H_{u}+h_{i}^{n} n_{i}^{c} n_{i}^{c} S,
$$

where we have used $S U(5)$ notation for ordinary quarks and leptons, $i=1,2,3$ counts the generations and $n^{c}$ denote the charge conjugate of right-handed neutrinos; $H_{u}, H_{d}$ and $S$ are the electroweak and $B-L$ symmetry breaking fields, respectively,

$$
\left\langle H_{u}\right\rangle=v_{u}, \quad\left\langle H_{d}\right\rangle=v_{d}, \quad\langle S\rangle=v_{B-L}
$$

The heavy Majorana neutrinos are given by $N_{i}=n_{i}+n_{i}^{c}$.

For the Yukawa couplings we shall use a representative model [12] with a FroggattNielsen $U(1)$ flavour symmetry, which is consistent with the observed hierarchical quark and lepton masses, thermal leptogenesis [12] as well as constraints from flavour changing processes [13]. Their order of magnitude is given by

$$
h_{i j} \propto \eta^{Q_{i}+Q_{j}}
$$

where the chiral $U(1)$ charges are listed in Table 1, the parameter $\eta \simeq 1 / \sqrt{300}$ is determined by the hierarchy of quark and lepton masses. 


\begin{tabular}{c|cccccccccccc}
\hline \hline$\psi_{i}$ & $\mathbf{1 0}_{3}$ & $\mathbf{1 0}_{2}$ & $\mathbf{1 0}_{1}$ & $\mathbf{5}_{3}^{*}$ & $\mathbf{5}_{2}^{*}$ & $\mathbf{5}_{1}^{*}$ & $n_{3}^{c}$ & $n_{2}^{c}$ & $n_{1}^{c}$ & $H_{u}$ & $H_{d}$ & $S$ \\
\hline$Q_{i}$ & 0 & 1 & 2 & 1 & 1 & 2 & 0 & 0 & 1 & 0 & 0 & 0 \\
\hline \hline
\end{tabular}

Table 1: Chiral $U(1)$ charges. $\mathbf{1 0}=\left(q, u^{c}, e^{c}\right), \mathbf{5}^{*}=\left(d^{c}, l\right)$.

Using the seesaw mass relation and the estimate $\bar{m}_{\nu}=\sqrt{m_{3} m_{2}} \sim 0.01 \mathrm{eV}$, successful thermal leptogenesis determines the mass of the lightest heavy Majorana neutrino, $M_{1}=$ $10^{10} \mathrm{GeV}[12]$. From

$$
M_{1}=h_{1}^{n} v_{B-L} \simeq \eta^{2} v_{B-L}
$$

one then obtains $v_{B-L} \simeq 3 \times 10^{12} \mathrm{GeV}$. The masses of the other heavy neutrinos coincide with the $B-L$ breaking scale,

$$
M_{2} \simeq M_{3} \simeq v_{B-L} \simeq 3 \times 10^{12} \mathrm{GeV}
$$

For simplicity, we shall only consider the Higgs boson of $B-L$ symmetry breaking in the following 2 . The Higgs potential relates the $S$ mass to the energy density of the unbroken phase,

$$
m_{S}^{2}=\lambda v_{B-L}^{2}, \quad \rho_{0}=\frac{1}{4} \lambda v_{B-L}^{4} .
$$

We assume the Higgs coupling $\lambda$ to be $\mathcal{O}(1)$, such that the Higgs mass lies in the range

$$
2 M_{2,3}>m_{S}>2 M_{1}
$$

The $U(1)$ flavour symmetry also determines the decay rates of the heavy Majorana neutrinos $N_{1}, N_{2,3}$ and of the Higgs boson $S$,

$$
\begin{aligned}
\Gamma_{N_{1}}^{0} & \simeq \frac{\eta^{4}}{8 \pi} M_{1}, \\
\Gamma_{N_{2,3}}^{0} & \simeq \frac{\eta^{2}}{8 \pi} M_{2,3}, \\
\Gamma_{S}^{0} & =\frac{\left(h_{1}^{n}\right)^{2}}{16 \pi} m_{S}\left[1-\left(2 M_{1} / m_{S}\right)^{2}\right]^{3 / 2} \simeq \frac{\eta^{4}}{16 \pi} m_{S} .
\end{aligned}
$$

Note that these estimates for the decay widths have a considerable uncertainty since the Yukawa couplings, which enter quadratically, are only known up to factors $\mathcal{O}(1)$ [12].

\footnotetext{
${ }^{2}$ The supersymmetric Higgs mechanism for $B-L$ breaking will be discussed in a forthcoming publication [14].
} 
For instance, since $M_{1}$ and $\Gamma_{N_{1}}^{0}$ involve different Yukawa couplings, the ratio $\Gamma_{N_{1}}^{0} / M_{1}$ can easily vary by two orders of magnitude. Since this ratio will be important in the following phenomenological analysis, we shall parametrize this uncertainty explicitly by the effective neutrino mass $\widetilde{m}_{1}$ introduced in Eq. (2).

A crucial ingredient of leptogenesis are the $C P$ asymmetries in heavy Majorana neutrinos decays [15,16],

$$
\epsilon_{i}=\frac{1}{8 \pi} \frac{1}{\left(h^{\nu \dagger} h^{\nu}\right)_{i i}} \sum_{j \neq i} \operatorname{Im}\left[\left(h^{\nu \dagger} h^{\nu}\right)_{i j}^{2}\right] F\left(\frac{M_{j}}{M_{i}}\right),
$$

from which we obtain

$$
\epsilon_{1} \sim 0.1 \eta^{4} \sim 10^{-6}, \quad \epsilon_{2,3} \simeq 0.1 \eta^{2} \sim 3 \times 10^{-4} .
$$

These asymmetries determine the lepton asymmetry which is generated by decays and inverse decays of the heavy neutrinos. Note that for $M_{1} \simeq 10^{10} \mathrm{GeV}$, the estimate $\epsilon_{1} \sim 10^{-6}$ corresponds to the maximal possible $C P$ asymmetry [17]. For smaller heavy neutrino masses the asymmetry is reduced by the mass ratio, $\epsilon_{1} \sim 10^{-6} M_{1} / 10^{10} \mathrm{GeV}$.

The vacuum energy of a phase with unbroken $B-L$ symmetry can drive a period of inflation, which then rapidly ends due to the spinodal growth of long wave-length modes, a process known as 'tachyonic preheating' [18]. The true vacuum is reached at a time $t_{\mathrm{PH}}$ after inflation [19],

$$
\left.\left\langle S^{\dagger} S\right\rangle\right|_{t=t_{\mathrm{PH}}}=v_{B-L}^{2}, \quad t_{\mathrm{PH}} \simeq \frac{1}{2 m_{S}} \ln \left(\frac{32 \pi^{2}}{\lambda}\right) .
$$

During this phase transition the energy of the false vacuum is converted mostly into a nonrelativistic gas of $S$ bosons, with an admixture of heavy neutrino 33 whose contribution to the energy density is determined by their coupling to the Higgs field [19],

$$
r_{N_{i}}=\frac{\rho_{N_{i}}}{\rho_{0}} \simeq 1.5 \times 10^{-3} g_{N} \lambda f\left(\alpha_{i}, 0.8\right) ;
$$

here $g_{N}=2$ and $f(\alpha, \gamma)=\sqrt{\alpha^{2}+\gamma^{2}}-\gamma$ with $\alpha_{i}=h_{i}^{n} / \sqrt{\lambda}$. For the neutrinos $N_{2,3}$ one obtains

$$
r_{N_{2,3}} \simeq 1 \times 10^{-3}\left(h_{2}^{n}\right)^{2} \simeq 1 \times 10^{-3} .
$$

The fraction $r_{N_{1}}=\mathcal{O}\left(\eta^{4}\right)$ and it is therefore negligible.

\footnotetext{
${ }^{3}$ For simplicity, we neglect the contribution of scalar neutrinos ; also in thermal leptogenesis their effect on the final baryon asymmetry is known to be small [20].
} 


\section{Reheating, baryon asymmetry and gravitino dark matter from heavy neutrino decays}

We are now ready to study the time evolution of the initial state produced by tachyonic preheating. First, the dominant contribution to the energy density is the nonrelativistic gas of $S$ bosons (cf. Eq. (19)) ),

$$
\rho_{S}(t)=\frac{1}{a^{3}(t)}\left(1-2 r_{N_{2}}\right) \rho_{0}
$$

where $a(t)$ is the scale factor of the Friedman universe which is normalized by setting $a\left(t_{\mathrm{PH}}\right)=1$. It is convenient to introduce the comoving number densities for all particle species $X$,

$$
N_{X}(t)=a^{3}(t) n_{X}(t)
$$

At $t \sim t_{2}=1 / \Gamma_{N_{2,3}}^{0}$ the heavy neutrinos $N_{2}$ and $N_{3}$ decay, producing the initial radiation density 4

$$
\rho_{R}\left(t_{2}\right)=\frac{2 r_{N_{2}} \rho_{0}}{a^{3}\left(t_{2}\right)}
$$

with temperature

$$
T\left(t_{2}\right)=\left(\frac{30}{\pi^{2} g_{*}} \frac{2 r_{N_{2}} \rho_{0}}{a^{3}\left(t_{2}\right)}\right)^{1 / 4} .
$$

The out-of-equilibrium decay of $N_{2}$ and $N_{3}$ also produces a $B-L$ asymmetry 5 . The corresponding comoving number density is given by

$$
N_{B-L}\left(t_{2}\right)=\epsilon_{2} N_{N_{2}}\left(t_{2}\right)+\epsilon_{3} N_{N_{3}}\left(t_{2}\right) \simeq 0.2 \eta^{2} N_{N_{2}}\left(t_{\mathrm{PH}}\right)
$$

The scale factor $a(t)$ has to be determined by solving the Friedman equation. For a flat universe and constant equation of state $\omega=p / \rho$ between some initial time $t_{0}$ and time $t$, one has

$$
a(t)=a\left(t_{0}\right)\left[1+\frac{3}{2}(1+\omega)\left(\frac{8 \pi}{3 M_{P}^{2}} \rho_{\text {tot }}\left(t_{0}\right)\right)^{1 / 2}\left(t-t_{0}\right)\right]^{\frac{2}{3(1+\omega)}}
$$

\footnotetext{
${ }^{4}$ Since $t_{2}$ is very small compared to the time scales relevant for leptogenesis, we approximate the onset of radiation by a step function.

${ }^{5}$ In $[19$ only the initial $B-L$ asymmetry from tachyonic preheating is taken into account.
} 
One expects that until the decay of the $S$ bosons at $t \sim t_{S}=1 / \Gamma_{S}^{0}$ the system is mostly nonrelativistic and that it becomes relativistic at later times. We have checked numerically that this is indeed the case with effective equation-of-state parameters $3\left(1+\omega_{\mathrm{PH}}\right) \simeq 3.3$ for $t_{\mathrm{PH}}<t \leq t_{S}$ and $3\left(1+\omega_{S}\right) \simeq 4.0$ for $t>t_{S}$, respectively.

The thermal part of the plasma due to $N_{2}$ and $N_{3}$ decays produces also $N_{1}$ neutrinos. Their comoving number density $N_{1}^{T}$ satisfies the familiar Boltzmann equation

$$
a H \frac{d}{d a} N_{N_{1}}^{T}=-\Gamma_{N_{1}}\left(N_{N_{1}}^{T}-N_{N_{1}}^{\mathrm{eq}}\right) ;
$$

here $H=\dot{a} / a$ is the Hubble parameter, and the thermal width

$$
\Gamma_{N_{1}}=\Gamma_{N_{1}}^{0} \frac{K_{1}(z)}{K_{2}(z)}, \quad z=\frac{M_{1}}{T},
$$

where $K_{1}$ and $K_{2}$ are modified Bessel functions. Note that in deriving Eq. (26) one assumes kinetic equilibrium for the thermally produced $N_{1}$ neutrinos.

$N_{1}$ neutrinos are also produced in $S$ decays. These neutrinos are relativistic, but not in kinetic equilibrium. They are produced with energy $m_{S} / 2$ which is then redshifted with increasing scale factor. We take this into account by solving the Boltzmann equations for the $S$ and $N_{1}$ distribution functions $\left(E_{N_{1}}=\sqrt{p^{2}+M_{1}^{2}}\right)$,

$$
\begin{aligned}
\left(\frac{\partial}{\partial t}-H p \frac{\partial}{\partial p}\right) f_{S}(t, p)= & -\frac{m_{S}}{E_{S}} \Gamma_{S}^{0} f_{S}(t, p) \\
\left(\frac{\partial}{\partial t}-H p \frac{\partial}{\partial p}\right) f_{N_{1}}^{S}(t, p)= & -\frac{M_{1}}{E_{N_{1}}} \Gamma_{N_{1}}^{0} f_{N_{1}}^{S}(t, p) \\
& +\frac{2 \pi^{2} n_{S} \Gamma_{S}^{0}}{E_{N_{1}}^{2}}\left[1-\left(2 M_{1} / m_{S}\right)^{2}\right]^{-1 / 2} \delta\left(E_{N_{1}}-m_{S} / 2\right) .
\end{aligned}
$$

A straightforward calculation yields for the distribution functions

$$
\begin{aligned}
f_{S}(t, p)= & \frac{2 \pi^{2} N_{S}\left(t_{2}\right)}{k^{2}} \delta(k) e^{-\Gamma_{S}^{0}\left(t-t_{2}\right)}, \quad p=\frac{k}{a(t)} \\
f_{N_{1}}^{S}(t, p)= & \frac{1}{a^{3}} \frac{4 \pi^{2} \Gamma_{S}^{0}}{m_{S}}\left(1-\left(2 M_{1} / m_{S}\right)^{2}\right)^{-\frac{1}{2}} \int_{t_{2}}^{t} d t^{\prime}\left[\frac{a}{a^{\prime}} \delta\left(E_{N_{1}}-\mathcal{E}_{N_{1}}\left(t^{\prime}, t\right)\right)\right. \\
& \left.\times \mathcal{E}_{N_{1}}^{-1}\left(t^{\prime}, t\right) N_{S}\left(t^{\prime}\right) \exp \left(-M_{1} \Gamma_{N_{1}}^{0} \int_{t^{\prime}}^{t} d t^{\prime \prime} \mathcal{E}_{N_{1}}^{-1}\left(t^{\prime}, t^{\prime \prime}\right)\right)\right],
\end{aligned}
$$

where $\mathcal{E}_{N_{1}}\left(t^{\prime}, t\right)$ is the redshifted energy at time $t$ of an $N_{1}$ neutrino produced in $S$ decay at time $t^{\prime}$ with energy $m_{S} / 2$,

$$
\mathcal{E}_{N_{1}}\left(t^{\prime}, t\right)=\frac{m_{S}}{2} \frac{a^{\prime}}{a}\left[1+\left(\left(\frac{a}{a^{\prime}}\right)^{2}-1\right)\left(\frac{2 M_{1}}{m_{S}}\right)^{2}\right]^{\frac{1}{2}} .
$$


The corresponding comoving number densities are easily obtained from

$$
N_{X}(t)=a^{3} g_{X} \int \frac{d^{3} p}{(2 \pi)^{3}} f_{X}(t, p)
$$

where $X=S, N_{1}$.

The number densities of thermally and nonthermally produced $N_{1}$ neutrinos enter as source terms in the Boltzmann equation for the $B-L$ asymmetry, which reads

$$
a H \frac{d}{d a} N_{B-L}=\epsilon_{1} \Gamma_{N_{1}}\left(N_{N_{1}}^{T}-N_{N_{1}}^{\mathrm{eq}}\right)-\frac{N_{N_{1}}^{\mathrm{eq}}}{2 N_{L}^{\mathrm{eq}}} \Gamma_{N_{1}} N_{B-L}+\epsilon_{1} \Gamma_{N_{1}}^{0} \widetilde{N}_{N_{1}}^{S},
$$

with the nonthermal contribution

$$
\widetilde{N}_{N_{1}}^{S}(t)=\int_{t_{2}}^{t} d t^{\prime} \frac{d N_{N_{1}}^{S}\left(t^{\prime}\right)}{d t^{\prime}} \frac{M_{1}}{\mathcal{E}_{N_{1}}\left(t^{\prime}, t\right)},
$$

which includes the relativistic correction factor for the decays of $N_{1}$ as function of time.

In addition to the baryon asymmetry we are interested in thermal production of gravitinos which is governed by the Boltzmann equation

$$
a H \frac{d}{d a} N_{\tilde{G}}=a^{3} \mathcal{C}_{\tilde{G}}(T)
$$

with the dominant QCD collision term in the supersymmetric Standard Model [7,8]

$$
\mathcal{C}_{\tilde{G}}(T)=\left(1+\frac{m_{\tilde{g}}^{2}}{3 m_{\tilde{G}}^{2}}\right) \frac{54 \zeta(3) g_{s}(T)^{2}}{\pi^{2} M_{P}} T^{6}\left[\ln \left(\frac{T^{2}}{m_{g}(T)^{2}}\right)+0.8846\right] ;
$$

here $g_{s}\left(10^{10} \mathrm{GeV}\right)=0.85$ is the QCD gauge coupling, and $m_{g}(T)=\sqrt{3 / 2} g_{s}(T) T$ is the plasma mass of the gluon.

Finally, we need the temperature as function of the scale factor in order to compute the collision term for gravitino production and the equilibrium number density of the $N_{1}$ neutrinos. The covariant energy conservation connects the thermal contributions to energy density and pressure with the nonthermal contributions,

$$
\frac{d}{d t}\left(\rho_{R}+\rho_{N_{1}}^{T}+\rho_{S}+\rho_{N_{1}}^{S}\right)+3 H\left(\rho_{R}+\rho_{N_{1}}^{T}+\rho_{S}+\rho_{N_{1}}^{S}+p_{R}+p_{N_{1}}^{T}+p_{N_{1}}^{S}\right)=0 .
$$

The quantities $\rho_{S}, \rho_{N_{1}}^{S}$ and $p_{N_{1}}^{S}$ are easily obtained from the corresponding distribution functions; energy and pressure of the thermally produced $N_{1}$ neutrinos are given in terms of the number density,

$$
a^{3} p_{N_{1}}^{T}=N_{N_{1}}^{T} T, \quad a^{3} \rho_{N_{1}}^{T}=T^{2} \frac{\partial}{\partial T} N_{N_{1}}^{T}
$$

Together with the Boltzmann equations (26), (34) and (36) we now have a complete system of first-order differential equations which determine the time evolution of the initial state, in particular the generation of baryon asymmetry and gravitino abundance. 


\section{Results and discussion}

We have numerically solved the Boltzmann equations derived in the previous section. In the following we describe the solution for a representative choice of neutrino masses, $C P$ asymmetrie 6 and superparticle masses: $\widetilde{m}_{1}=10^{-3} \mathrm{eV}, M_{1}=10^{10} \mathrm{GeV}, \epsilon_{1}=10^{-6}$, $\epsilon_{2,3}=-3 \times 10^{-4}$, and $m_{\tilde{G}}=100 \mathrm{GeV}, m_{\tilde{g}}=800 \mathrm{GeV}$. Technical details of the solution and a systematic study of the parameter space compatible with leptogenesis and gravitino dark matter will be presented in a forthcoming publication [14.

The various components of the energy density and the comoving number densities are compared as functions of the scale factor $a$ in Fig. 1 and Fig. 2, respectively. Immediately after the end of tachyonic preheating the decay of $N_{2}$ and $N_{3}$ generates radiation, an initial $B-L$ asymmetry and subsequently a thermal abundance of $N_{1}$ neutrinos. At $a \sim 2$, the $S$ bosons decay. The produced $N_{1}$ neutrinos are relativistic, but nonthermal. At $a \sim 150$, the thermal $N_{1}$ neutrinos reach thermal equilibrium, the initial $B-L$ asymmetry is washed out, a new $B-L$ asymmetry and the dominant part of radiation are produced in decays of the nonthermal $N_{1}^{S}$ neutrinos. Furthermore, gravitinos are continuously produced from the thermal bath. Around $a \sim 1000$ the final $B-L$ asymmetry and the gravitino number density are reached.

The present value of baryon asymmetry is obtained from

$$
\eta_{B}=\frac{n_{B}^{0}}{n_{\gamma}^{0}}=\left.c_{\mathrm{sph}} \frac{g_{*}^{0}}{g_{*}} \frac{N_{B-L}}{N_{\gamma}}\right|_{a_{f}},
$$

where $a_{f} \sim 10^{4}$ is a scale factor after leptogenesis is completed; in the supersymmetric Standard Model the sphaleron conversion factor $c_{\mathrm{sph}}=8 / 23$, the effective numbers of degrees of freedom at high and low temperatures are $g_{*}=915 / 4$ and $g_{*}^{0}=43 / 11$, respectively, and the number density of photons is $N_{\gamma}=a^{3} g_{\gamma} \zeta(3) / \pi^{2} T^{3}$. The corresponding expression for the present gravitino abundance is given by

$$
\Omega_{\tilde{G}}=\left.\frac{m_{\tilde{G}} n_{\gamma}^{0}}{\rho_{c}^{0}} \frac{g_{*}^{0}}{g_{*}} \frac{N_{\tilde{G}}}{N_{\gamma}}\right|_{a_{f}},
$$

where $\rho_{c}^{0}=1.052 \times 10^{-5} h^{2} \mathrm{GeVcm}^{-3}$ is the critical density. For our choice of parameters we obtain

$$
\eta_{B}=1.6 \times 10^{-7}, \quad \Omega_{\tilde{G}} h^{2}=0.11 .
$$

\footnotetext{
${ }^{6}$ We have chosen opposite signs for the $C P$ asymmetries $\epsilon_{1}$ and $\epsilon_{2,3}$, so that one can distinguish their contribution to the final $B-L$ asymmetry.
} 


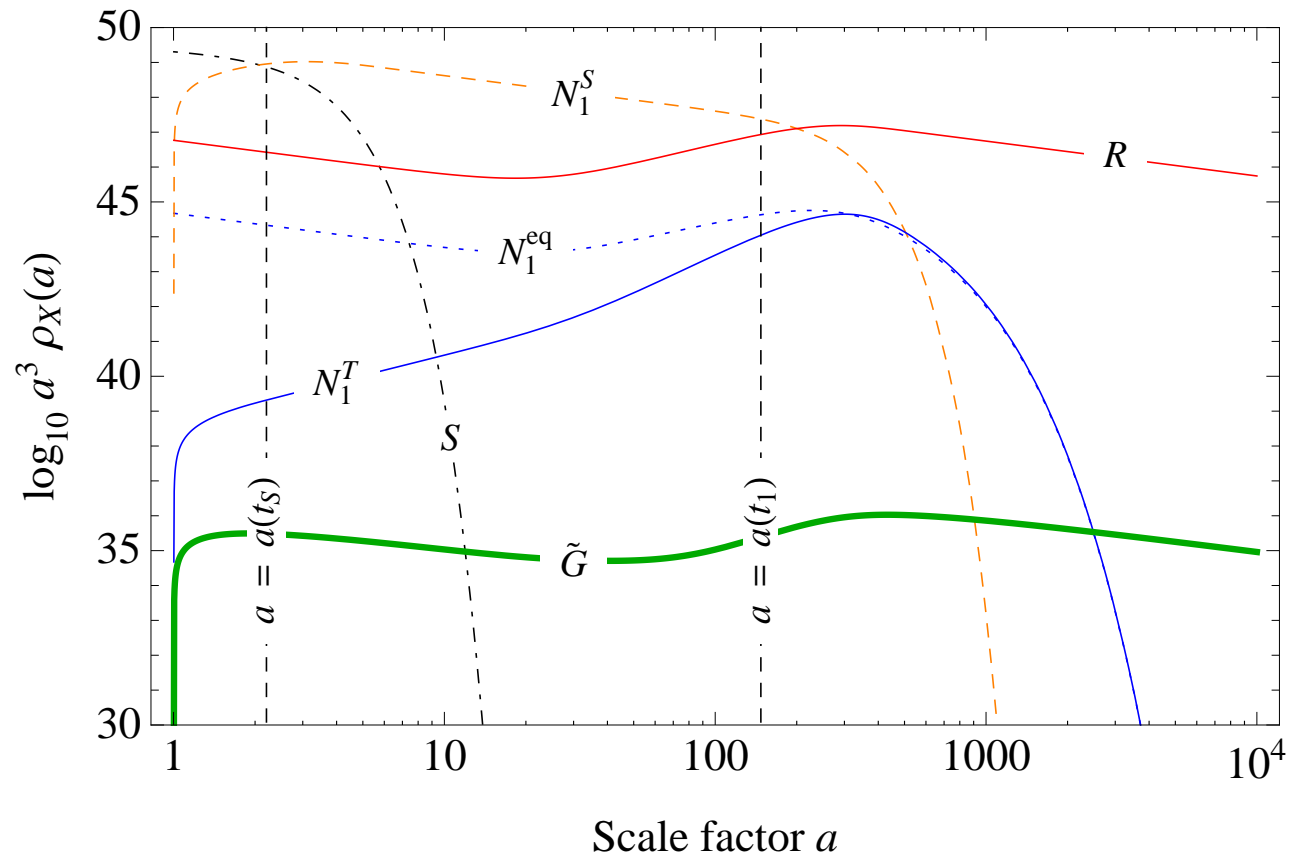

Figure 1: Comoving energy densities for $S$ bosons, radiation $(R), N_{1}$ neutrinos produced in $S$ decays $\left(N_{1}^{S}\right), N_{1}$ neutrinos in equilibrium ( $N_{1}^{\mathrm{eq}}$, for comparison), thermally produced $N_{1}$ neutrinos $\left(N_{1}^{T}\right)$ and gravitinos $(\tilde{G})$ as functions of the scale factor $a$.

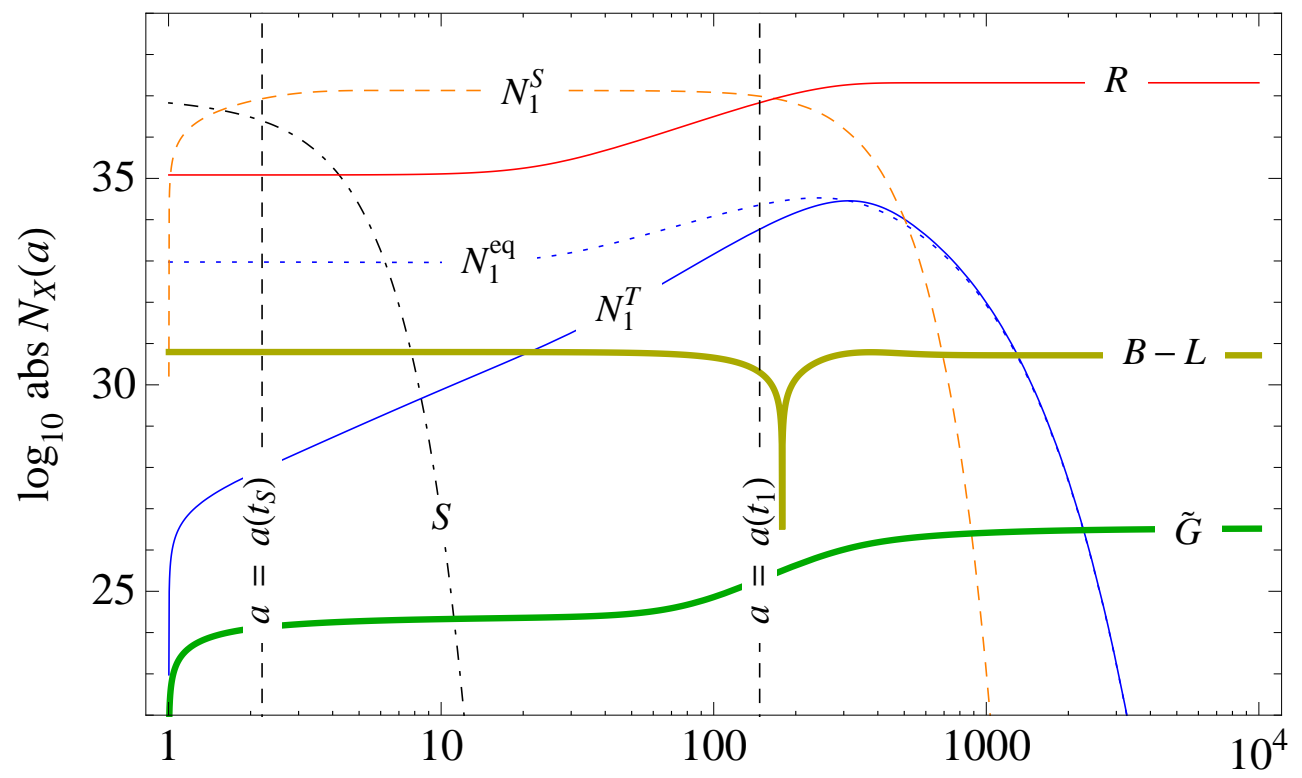

Scale factor $a$

Figure 2: Comoving number densities for $S$ bosons, $B-L$ charge $(B-L) N_{1}$ neutrinos produced in $S$ decays $\left(N_{1}^{S}\right), N_{1}$ neutrinos in equilibrium ( $N_{1}^{\mathrm{eq}}$, for comparison), thermally produced $N_{1}$ neutrinos $\left(N_{1}^{T}\right)$ and gravitinos $(\tilde{G})$ as functions of the scale factor $a$. 


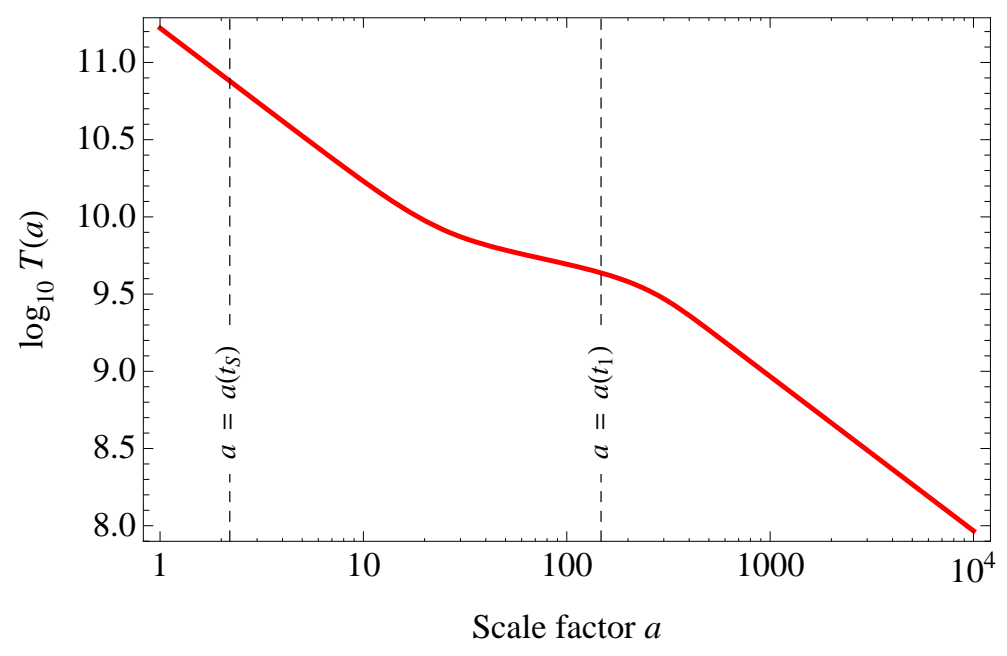

Figure 3: Radiation temperature as function of the scale factor $a$.

The gravitino abundance is close to the observed value for $\Omega_{\mathrm{DM}}$. The calculated baryon asymmetry is about two orders of magnitude larger than the observed one, $\eta_{B}^{\mathrm{CMB}} \simeq$ $6.2 \times 10^{-10}$ [10], which is consistent since we have used the maximal $C P$ asymmetry.

The temperature of radiation is shown in Fig. 3 as function of the scale factor. It decreases like $T \propto 1 / a$ except for an approximate plateau around $T_{L} \simeq 5 \times 10^{9} \mathrm{GeV}$ at $a \sim 100$, when the $N_{1}$ neutrinos decay. Note that the temperature $T_{L}$ agrees with the reheating temperature $T_{R}$ estimated in Eq. (4) up to a factor of two. Inserting the temperature $T_{L}$ in the estimate Eq. (1) for the gravitino abundance, one obtains agreement with the result of the full calculation given in Eq. (42). For such large reheating temperatures nonthermal production of gravitinos is usually negligible [21].

It is instructive to compare the calculated baryon asymmetry with two extreme cases, namely thermal leptogenesis and the rapid conversion of a gas of nonrelativistic $N_{1}$ neutrinos dominating the energy density of the universe. For thermal leptogenesis one obtains for our choice of parameters (cf. [1] )

$$
\eta_{B}^{\text {thermal }}=\frac{3}{4} \frac{g_{*}^{0}}{g_{*}} c_{\mathrm{sph}} \epsilon_{1} \kappa_{f}\left(\widetilde{m}_{1}\right) \simeq 5 \times 10^{-10}
$$

where we have used $c_{\mathrm{sph}}=8 / 23$ and $\kappa_{f}\left(10^{-3} \mathrm{eV}\right) \simeq 0.1$ for the efficiency factor. In the case of rapid conversion, energy conservation yields (cf. [22])

$$
\eta_{B}^{\text {rapid }} \simeq 7 \frac{3}{4} c_{\mathrm{sph}} \epsilon_{1} \frac{T_{L}}{M_{1}} \simeq 9 \times 10^{-7}
$$


which is closer to the result of our calculation Eq. (42). Varying the parameters $\widetilde{m}_{1}$ and $M_{1}$ one can interpolate between thermal and nonthermal leptogenesis. The effective neutrino mass $\widetilde{m}_{1}$ is closely related to the smallest neutrino mass $m_{1}$ and therefore to the absolute neutrino mass scale. Via its effect on the reheating temperature and gravitino dark matter, the absolute neutrino mass scale is thus related to the gravitino mass.

The case of rapid conversion is also realized in leptogenesis from inflaton decays [23]. Here the reheating temperature is determined by the decay width of the inflaton. One can then have $T_{R} \ll M_{1}$ and in this way avoid overproduction of gravitinos. For larger inflaton decay widths reheating temperatures $T_{R} \sim M_{1}$ can be reached, associated with larger baryon asymmetries [24], similar to the situation described in this paper.

We have shown that reheating the universe by heavy Majorana neutrino decays can simultaneously explain the cosmological baryon asymmetry and the observed dark matter

abundance in terms of thermally produced gravitinos. Starting from an initial state of unbroken $B-L$ symmetry, tachyonic preheating leads to an interplay of nonthermal and thermal leptogenesis, which is controlled by the heavy neutrino decay widths. Open questions concern the connection with models of inflation and tachyonic preheating in supersymmetric theories.

\section{References}

[1] M. Fukugita and T. Yanagida, Phys. Lett. B 174, 45 (1986).

[2] W. Buchmuller, R. D. Peccei and T. Yanagida, Ann. Rev. Nucl. Part. Sci. 55 (2005) 311 [hep-ph/0502169]; S. Davidson, E. Nardi and Y. Nir, Phys. Rept. 466 (2008) 105 [0802.2962 [hep-ph]].

[3] S. Weinberg, Phys. Rev. Lett. 48 (1982) 1303.

[4] J. R. Ellis, D. V. Nanopoulos and S. Sarkar, Nucl. Phys. B 259 (1985) 175.

[5] M. Kawasaki, K. Kohri and T. Moroi, Phys. Lett. B 625, 7 (2005) astro-ph/0402490; Phys. Rev. D 71, 083502 (2005) astro-ph/0408426;

K. Jedamzik, Phys. Rev. D 74, 103509 (2006) [hep-ph/0604251].

[6] M. Bolz, W. Buchmuller and M. Plumacher, Phys. Lett. B 443 (1998) 209 hep-ph/9809381.

[7] M. Bolz, A. Brandenburg and W. Buchmuller, Nucl. Phys. B 606, 518 (2001) [Erratum-ibid. B 790, 336 (2008)] [hep-ph/0012052]. 
[8] J. Pradler and F. D. Steffen, Phys. Rev. D 75, 023509 (2007). hep-ph/0608344.

[9] V. S. Rychkov and A. Strumia, Phys. Rev. D 75, 075011 (2007). hep-ph/0701104.

[10] E. Komatsu et al., arXiv:1001.4538 [astro-ph.CO].

[11] W. Buchmuller, P. Di Bari and M. Plumacher, Nucl. Phys. B 665 (2003) 445 hep-ph/0302092.

[12] W. Buchmüller and T. Yanagida, Phys. Lett. B 445 (1999) 399 [hep-ph/9810308].

[13] W. Buchmuller, D. Delepine and L. T. Handoko, Nucl. Phys. B 576 (2000) 445 hep-ph/9912317.

[14] W. Buchmuller, K. Schmitz and G. Vertongen, in preparation

[15] L. Covi, E. Roulet and F. Vissani, Phys. Lett. B 384 (1996) 169 [hep-ph/9605319].

[16] W. Buchmuller and M. Plumacher, Phys. Lett. B 431 (1998) 354 hep-ph/9710460.

[17] S. Davidson and A. Ibarra, Phys. Lett. B 535 (2002) 25 hep-ph/0202239.

[18] G. N. Felder, J. Garcia-Bellido, P. B. Greene, L. Kofman, A. D. Linde and I. Tkachev, Phys. Rev. Lett. 87 (2001) 011601 hep-ph/0012142]; G. N. Felder, L. Kofman and A. D. Linde, Phys. Rev. D 64 (2001) 123517 [hep-th/0106179].

[19] J. Garcia-Bellido and E. Ruiz Morales, Phys. Lett. B 536 (2002) 193 hep-ph/0109230.

[20] M. Plumacher, Nucl. Phys. B 530 (1998) 207 hep-ph/9704231].

[21] For a recent discussion and references, see K. Nakayama, F. Takahashi and T. T. Yanagida, 1007.5152 [hep-ph].

[22] E. W. Kolb and M. S. Turner, The Early universe, Front. Phys. 69 (1990) 1.

[23] T. Asaka, K. Hamaguchi, M. Kawasaki and T. Yanagida, Phys. Lett. B 464 (1999) 12 [hep-ph/9906366]; Phys. Rev. D 61 (2000) 083512 [hep-ph/9907559].

[24] F. Hahn-Woernle and M. Plumacher, Nucl. Phys. B 806 (2009) 68 [0801.3972 [hep$\mathrm{ph}]$. 